\title{
Constraints on non-resonant photon-axion conversion from the Planck satellite data
}

\author{
Suvodip Mukherjee, ${ }^{a, b, c}$ Rishi Khatri ${ }^{d}$ and Benjamin D. \\ Wandelt $\mathbf{t}^{a, b, c, e}$ \\ ${ }^{a}$ Center for Computational Astrophysics, Flatiron Institute, 162 5th Avenue, 10010, New York, NY, \\ USA \\ ${ }^{b}$ Institut d'Astrophysique de Paris \\ 98bis Boulevard Arago, 75014 Paris, France \\ ${ }^{c}$ Sorbonne Universites, Institut Lagrange de Paris \\ 98 bis Boulevard Arago, 75014 Paris, France \\ ${ }^{d}$ Department of Theoretical Physics, Tata Institute of Fundamental Research \\ Homi Bhabha Road, Mumbai, 400005, India \\ ${ }^{e}$ Dept. of Astrophysical Sciences, Princeton University, Princeton, NJ 08544, USA \\ E-mail: mukherje@iap.fr, khatri@theory.tifr.res.in, bwandelt@iap.fr
}

\begin{abstract}
The non-resonant conversion of Cosmic Microwave Background (CMB) photons into scalar as well as light pseudoscalar particles such as axion-like particles (ALPs) in the presence of turbulent magnetic fields can cause a unique, spatially fluctuating spectral distortion in the CMB. We use the publicly available Planck temperature maps for the frequency channels $(70-545 \mathrm{GHz})$ to obtain the first ALP distortion map using $45 \%$ clean part of the sky. The $95^{\text {th }}$ percentile upper limit on the RMS fluctuation of ALP distortions from the cleanest part of the CMB sky at 15 arcmin angular resolution is $18.5 \times 10^{-6}$. The RMS fluctuation in the distortion map is also consistent with different combinations of frequency channels and sky-fractions.
\end{abstract}

Keywords: Axion, Cosmic Microwave Background, Spectral Distortions 


\section{Contents}

1 Introduction $\quad 1$

2 Mechanism 2

3 Component separation for the axion spectrum 3

4 Results $\quad 5$

5 Conclusion $\quad 6$

\section{Introduction}

Distortions in the blackbody spectrum of Cosmic Microwave Background (CMB) are expected from several physical effects like thermal Sunyaev-Zeldovich ( $y$-type distortion) in clusters of galaxies, Silk damping, axions, recombination lines, dark-matter annihilations, dark matter decay etc. [1-18]. These effects span a wide range of redshifts from $z=2 \times 10^{6}$ to $z=0$ and are an excellent probe of both the early and the late time cosmic evolution. The spectral distortions can be spatially isotropic, affecting only the CMB monopole intensity, or anisotropic with spatial fluctuations in the sky. The CMB spectral distortions arising from photon-ALPs conversion can also be polarized due to the resonant conversion in the large scale coherent magnetic field such as that of Milky Way [18] and galaxy clusters. The non-resonant photon-ALPs conversion due to the small scale magnetic field of Milky Way, galaxy clusters and voids can produce unpolarized spectral distortion in CMB [18].

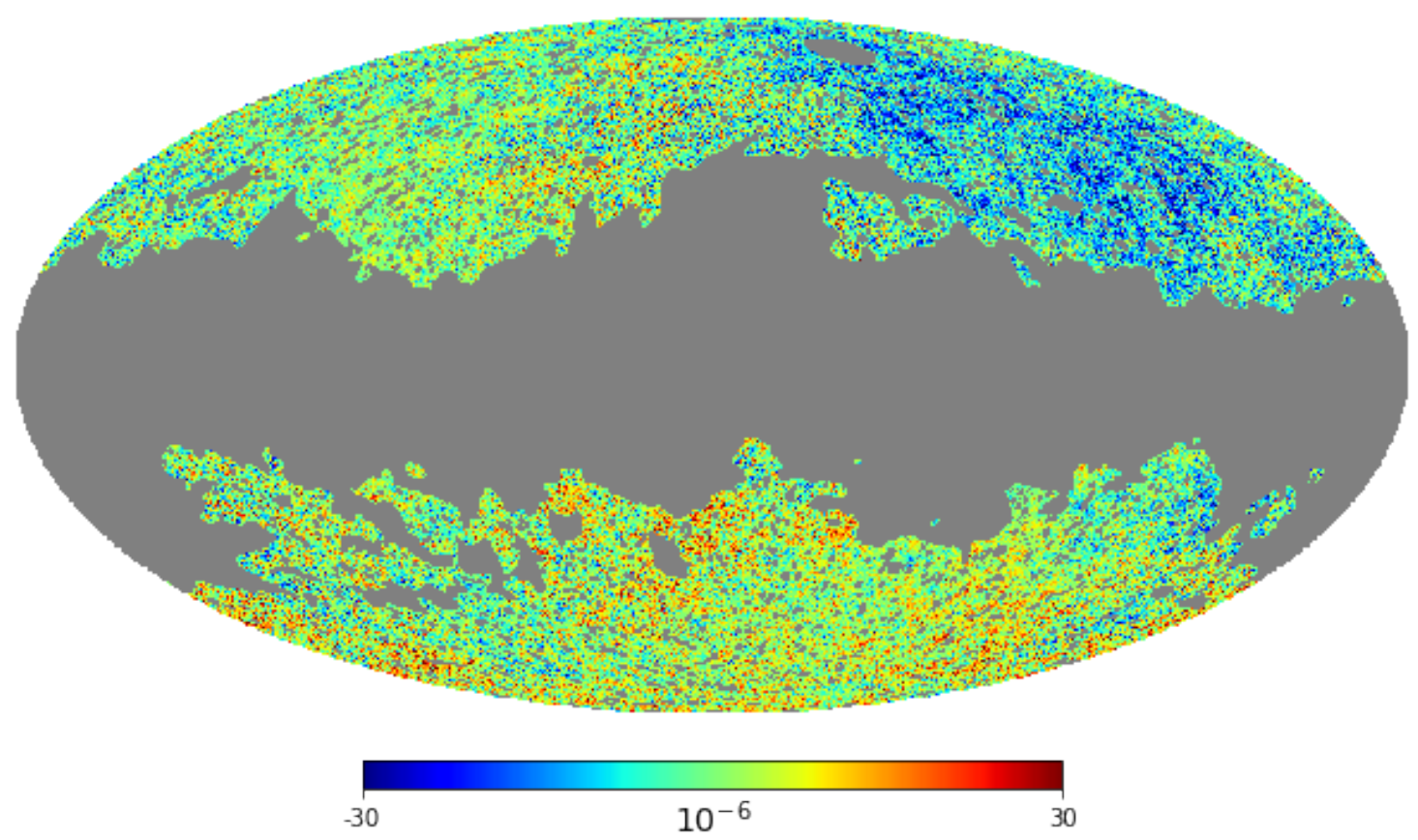

Figure 1: Internal Linear Combination Map of Axion Spectral Distortion (ASD) amplitude $P(\gamma \rightarrow a)$ (See Eq. (2.5)) for $f_{s k y}=0.45$ obtained from the six Planck-2015 temperature maps from 70 to 545 GHz. The details are provided in the results section. 
The most stringent full sky observational constraints on the monopole part of the CMB spectral distortions come from the Cosmic Background Explorer-Far Infrared Absolute Spectrophotometer (COBE-FIRAS) [19-22] with a $2 \sigma$ upper limit on the $y$-type distortion of $y \leq 15 \times 10^{-6}$ and on the $\mu$-type distortion of $\mu \leq 9 \times 10^{-5}$. The Planck experiment with its multiple channels and wide frequency coverage, has allowed us for the first time after COBE to study and constrain other types of spectral distortions near the peak of the CMB spectrum. Since Planck, unlike COBE-FIRAS, does not have an absolute calibrator, we can only study the spectral distortions which are anisotropic. The spatially fluctuating $y$-type spectral distortions have been measured in clusters [10-17], an upper and lower bound on the average distortions was obtained from the Planck data in [23] and an upper bound on the anisotropic $\mu$-type distortions from non-Gaussianity [24] was obtained from the Planck data in $[25]$.

In this paper, we study the spectral distortions that can originate from the non-resonant photon ALP conversion [18] (Axion Spectral Distortion (ASD)) in the presence of stochastic magnetic fields. This process can induce an unpolarized CMB spectral distortion signal that fluctuates spatially and has a spectral shape that is different from the other known spectral distortions. This new scheme enables to probe the existence of ALPs in nature over a vast range of masses $\left(m_{a}<10^{-11} \mathrm{eV}\right)$. ALPs are potential candidates for cold dark matter and are predicted by string theory scenarios [26]. Several ground based experiments such CAST [27], ALPS-II [28], MADMAX [29], ADMX [30], CASPER [31] are searching for ALPs. The method explored in this work is an independent method to detect ALPs using spatially varying spectral distortion signal in the blackbody spectrum of CMB. Using the frequency spectrum of the ASD signal, we obtain the ASD sky map (shown in Fig 1) using the Internal Linear Combination (ILC) component separation method [32] on multi-frequency Planck sky maps (70-545 GHz) smoothed to a common resolution of 15 arcmin.

The ASD signal depends strongly on the structure of the magnetic field and inhomogeneities in the electron density. We therefore need independent constraints on the 3-D structure of electron density and magnetic fields to translate the bounds on the ASD into constraints on Photon-ALPS coupling. We will use simple idealized models of magnetic field and electron density to derive joint bounds on the Photon-ALPs coupling, magnetic field and electron density. The polarized spectral distortions in the polarized sky maps from Planck can be used to provide a bound on the photon-axion coupling strength in a narrow mass range of ALPs, $10^{-13} \mathrm{eV} \leq m_{a} \leq 10^{-11} \mathrm{eV}$. We leave the analysis of polarized ASD for future work.

\section{Mechanism}

The conversion of the photons $\left(A_{x}, A_{y}\right)$ into axions $(a)$ in the presence of external magnetic field can be expressed by the differential equation [33-35]

$$
\left(\omega+\left(\begin{array}{ccc}
\Delta_{\mathrm{e}} & 0 & \Delta_{\gamma \mathrm{a}}^{x} \\
0 & \Delta_{\mathrm{e}} & \Delta_{\gamma \mathrm{a}}^{y} \\
\Delta_{\gamma \mathrm{a}}^{x} & \Delta_{\gamma \mathrm{a}}^{y} & \Delta_{\mathrm{a}}
\end{array}\right)+i \partial_{z}\right)\left(\begin{array}{c}
A_{x} \\
A_{y} \\
a
\end{array}\right)=0
$$

where, we have assumed that the photons are propagating in the z-direction and,

$$
\begin{aligned}
\left(\frac{\Delta_{\gamma \mathrm{a}}^{x, y}}{\mathrm{Mpc}^{-1}}\right) & \equiv \frac{g_{\gamma \mathrm{a}}\left|B_{x, y}\right|}{2}=15.2\left(\frac{g_{\gamma \mathrm{a}}}{10^{-11} \mathrm{Gev}^{-1}}\right)\left(\frac{B_{x, y}}{\mu \mathrm{G}}\right), \\
\left(\frac{\Delta_{\mathrm{a}}}{\mathrm{Mpc}^{-1}}\right) & \equiv-\frac{m_{a}^{2}}{2 \omega}=-1.9 \times 10^{4}\left(\frac{m_{\mathrm{a}}}{10^{-14} \mathrm{eV}}\right)\left(\frac{100 \mathrm{GHz}}{\nu}\right), \\
\left(\frac{\Delta_{\mathrm{e}}}{\mathrm{Mpc}^{-1}}\right) & \approx \frac{\omega_{\mathrm{p}}^{2}}{2 \omega}\left[-1+7.3 \times 10^{-3} \frac{n_{\mathrm{H}}}{n_{\mathrm{e}}}\left(\frac{\omega}{\mathrm{eV}}\right)^{2}\right] \\
& =-2.6 \times 10^{6}\left(\frac{n_{\mathrm{e}}}{10^{-5} \mathrm{~cm}^{-3}}\right)\left(\frac{100 \mathrm{GHz}}{\nu}\right),
\end{aligned}
$$

where $g_{\gamma \mathrm{a}}$ is the photon-ALP coupling strength, $\omega_{\mathrm{p}}=\left[4 \pi \alpha n_{\mathrm{e}} /\left(m_{\mathrm{e}}\right)\right]^{1 / 2}$ is the plasma frequency, $m_{e}$ is the mass of electron, $n_{e}$ is the electron density, $m_{a}$ is the mass of ALPs, $B_{x, y}$ is the magnetic field 
along the direction $x$ or $y$ and $n_{H}$ is the density of the hydrogen atoms. We neglect Faraday rotation. For homogeneous magnetic field and electron density, the above equation reduces to the simple form $[33-35]$

$$
P(\gamma \rightarrow a)=\frac{\left(\Delta_{\gamma \mathrm{a}} s\right)^{2}}{\left(\Delta_{\mathrm{osc}} s / 2\right)^{2}} \sin ^{2}\left(\Delta_{\mathrm{osc}} s / 2\right),
$$

where $\Delta_{\text {osc }}^{2}=\left(\Delta_{\mathrm{a}}-\Delta_{\mathrm{e}}\right)^{2}+4 \Delta_{\gamma \mathrm{a}}^{2}$ and $s$ is the length along the line of sight. The mixing angle $\theta$ can be defined as $\sin (2 \theta)=2 \Delta_{\gamma \mathrm{a}} / \Delta_{\text {osc }}$. For the case of inhomogeneous magnetic field and electron density, we need to solve Eq. (2.1) along the line of sight, to calculate the probability of conversion.

It is possible to obtain approximate analytical solutions for Milky Way, galaxy clusters and voids in the limit of stochastic magnetic fields with electron density changing slowly compared to the magnetic fields such that the adiabiticity parameter $\gamma_{a d} \equiv\left|\frac{\Delta_{\text {osc }}}{2 \nabla \theta}\right|<1[18]$

$$
\begin{aligned}
& P(\gamma \rightarrow a) \approx \frac{\Delta_{\gamma \mathrm{a}}^{2} R d}{2}=10^{-5}\left(\frac{g_{\gamma \mathrm{a}}}{10^{-9} \mathrm{GeV}^{-1}}\right)^{2}\left(\frac{B_{T}}{1 \mu \mathrm{G}}\right)^{2}\left(\frac{R}{10 \mathrm{kpc}}\right)\left(\frac{d}{10^{-3} \mathrm{pc}}\right) \text { for Milky Way, } \\
& \bar{P}(\gamma \rightarrow a) \approx \frac{\Delta_{\gamma \mathrm{a}}^{2} R_{\mathrm{V}} d}{2}=10^{-6}\left(\frac{g_{\gamma \mathrm{a}}}{10^{-10} \mathrm{GeV}^{-1}}\right)^{2}\left(\frac{B_{T}}{1 \mathrm{nG}}\right)^{2}\left(\frac{R_{\mathrm{V}}}{10 \mathrm{Mpc}}\right)\left(\frac{d}{10 \mathrm{pc}}\right) \text { for voids, } \\
& \bar{P}(\gamma \rightarrow a) \approx \frac{\Delta_{\gamma \mathrm{a}}^{2} R_{\mathrm{C}} d}{2}=10^{-5}\left(\frac{g_{\gamma \mathrm{a}}}{10^{-9} \mathrm{GeV}^{-1}}\right)^{2}\left(\frac{B_{T}}{0.1 \mu \mathrm{G}}\right)^{2}\left(\frac{R_{\mathrm{C}}}{10 \mathrm{Mpc}}\right)\left(\frac{d}{10^{-4} \mathrm{pc}}\right) \text { for galaxy clusters, }
\end{aligned}
$$

where $B_{T}$ is the magnetic field transverse to the line of sight in the domain of size $d$ and $R$ is the typical size of the region being considered $\left(R_{\mathrm{V}}\right.$ and $R_{\mathrm{C}}$ corresponds to the typical size considered for voids and galaxy clusters). In the above equation we have used the value of magnetic field which are consistent with the recent observations from synchrotron emission map and rotation measures [36, 37] and for galaxy clusters, the value of magnetic field are inferred from the Faraday rotation [38].

The above equation shows that the photon-axion coupling strength $g_{\gamma \mathrm{a}}$ is degenerate with astrophysical parameters like $B_{T}, R$ and $d$. The change in the intensity of the CMB is given by

$$
\begin{aligned}
\Delta I^{\gamma \mathrm{a}}(\nu, \hat{p}) & =P(\gamma \rightarrow a, \hat{p})\left(\frac{2 h \nu^{3}}{c^{2}}\right) \frac{1}{\left(e^{x}-1\right)}, \\
& =\alpha(\hat{p})\left(\frac{2 h \nu^{3}}{c^{2}}\right) \frac{1}{\left(e^{x}-1\right)},
\end{aligned}
$$

where, $x=h \nu /\left(k_{B} T_{C M B}\right), T_{C M B}=2.7255 \mathrm{~K}$ and $h, c$, and $k_{B}$ are Planck's constant, speed of light and Boltzmann constant respectively. In Eq. (2.5), $P(\gamma \rightarrow a, \hat{p}) \equiv \alpha(\hat{p})$ is the amplitude of the distortion along the direction denoted by $\hat{p}$. The direction dependence of $P(\gamma \rightarrow a, \hat{p})$ in the above equation arises due to the direction dependence of the magnetic field strength. All our results will be for this amplitude which varies over the sky while the shape of the distortion is fixed.

The spatially fluctuating spectral distortions of the CMB can be measured by experiments without an absolute calibrator but having multiple frequency channels, such as WMAP [39] and Planck [40].

\section{Component separation for the axion spectrum}

The Planck satellite measured the differential sky intensity in nine frequency channels covering the frequency range $30-857 \mathrm{GHz}$. The sky signal is a combination of several components including Galactic foregrounds (like synchrotron, free-free, AME, galactic dust), CMB, thermal Sunyaev-Zeldovich (tSZ) $[1,2]$, and Cosmic Infrared Background (CIB). A number of algorithms have been developed over the past decades to separate the observed sky signal into different components [32, 41-50].

In this analysis, we consider six frequency channels $(70,100,143,217,353,545 \mathrm{GHz})$ to obtain the sky-map for the ASD signal. Channels below $70 \mathrm{GHz}$ and above $545 \mathrm{GHz}$ are highly contaminated 


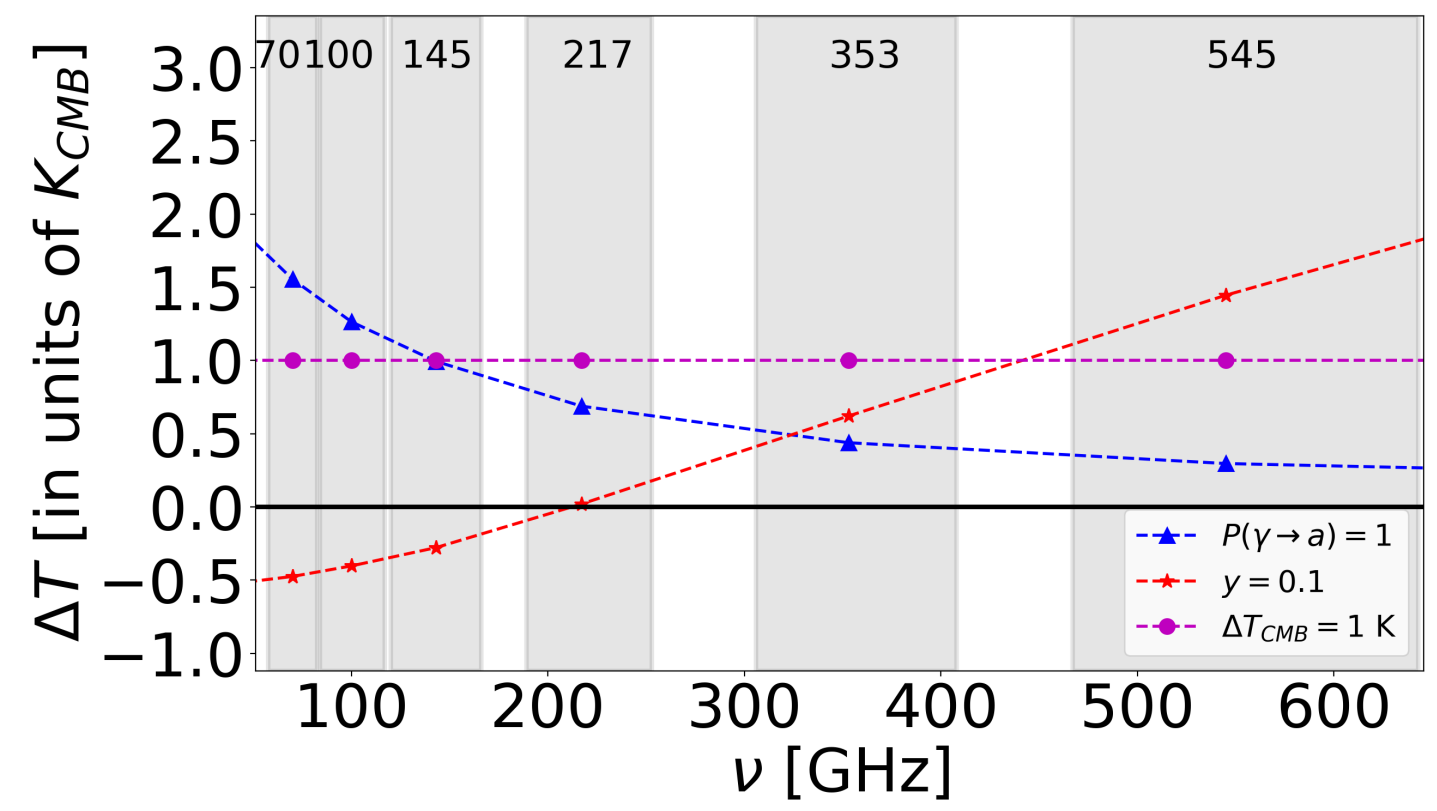

Figure 2: Frequency spectrum of the non-resonance photon-axion signal (in blue), $y$-type distortion for $y=0.1$ (in red) and CMB fluctuation for $\Delta T=1 K_{C M B}$ ( in magenta) are plotted in thermodynamic temperature units $\left(K_{C M B}\right)$ using Eq. (3.1). The grey bands indicate the frequency channels used in this analysis and the central frequency is mentioned in the top.

by synchrotron emission/AME, and dust respectively. So, we only consider these six channels in this analysis. These six frequency channels are also not completely clean and are dominated by foreground contaminations in the galactic plane. There are also point source contaminations of both galactic and extragalactic origin. We will use the ILC algorithm to separate the axion distortion from other cosmological and Galactic components $[46,51]$. In order to remove the worst Galactic and point source contamination, we apply a mask on the full sky map and use only partial sky in the analysis. We consider two different masks having usable sky-fraction $f_{\text {sky }}=27 \%$ and $45 \%$ [25], created specially to search for new spectral distortions in the Planck data. The masks are publicly available [52]. The $45 \%$ mask is shown in Fig. 1.

These masks remove the point source contaminations (tSZ, CO line emission) along with the most contaminated region of the Galaxy. The Planck frequency sky maps are in the CMB temperature units $\left(K_{C M B}\right)$ except for $545 \mathrm{GHz}$ map which has units of $\mathrm{MJy} / \mathrm{Sr}$ and which we also convert into $K_{C M B}$ units [53]. The different frequency channels also have a finite transmission bandwidth $\left(w_{\nu}\left(\nu^{\prime}\right)\right)$ [54]. So in order to extract the signal with a particular spectrum, we need to convert the spectrum from intensity to $K_{\mathrm{CMB}}$ units in the particular frequency band by integrating over the transmission function, using the relation [55]

$$
\Delta T^{\beta}(\nu)=\frac{\int w_{\nu}\left(\nu^{\prime}\right) I^{\beta}\left(\nu^{\prime}\right) d \nu^{\prime}}{\int w_{\nu}\left(\nu^{\prime}\right) I^{\prime p l}\left(\nu^{\prime}\right) d \nu^{\prime}}\left[\text { in units of } K_{C M B}\right]
$$

where, $I^{\prime p l}(\nu) \equiv \frac{\partial I^{p l}(\nu)}{\partial T}$ and $\beta \in\{\mathrm{CMB}, \mathrm{ASD}, \mathrm{y}$-type distortions (SZ), $\mu$-type $\}$. The bandpass corrected frequency spectrum of the ASD, CMB and SZ are shown in Fig. 2 along with the frequency bands used in the analysis.

The observed sky signal at different frequencies $\left(S_{\nu_{i}}\right)$ can be modeled in terms of the multiple components as

$$
\mathbf{S}(\hat{p})=\mathbf{A x}(\hat{p})+\mathbf{n}(\hat{p}),
$$


here $\mathbf{A}$ is the mixing matrix $\left[\mathbf{a}_{1}, \mathbf{a}_{2}, \ldots, \mathbf{a}_{\mathbf{M}}\right]$ with dimension $N \times M$, where $N$ is the number of frequency channels and $M$ is the number of components, $\mathbf{a}_{\mathbf{i}}$ is the spectrum of the $i^{\text {th }}$ component, $\mathbf{n}$ is the noise at pixel $\hat{p}$. The ILC solution for the axion signal with known spectrum $\mathbf{a}_{\gamma \mathbf{a}}$ is given by the linear combination of the input maps $[46,51]$

$$
\alpha=\hat{\mathbf{W}}_{\gamma a}^{T} \mathbf{S}(\hat{p}) .
$$

where, $\hat{\mathbf{W}}_{\gamma a}(\nu)=\mathbf{C}_{S}^{-1} \mathbf{a}_{\gamma a}\left(\mathbf{a}_{\gamma a}^{T} \mathbf{C}_{S}^{-1} \mathbf{a}_{\gamma a}\right)^{-1}$ and $\mathbf{C}_{S}=\left\langle\mathbf{S S}^{T}\right\rangle^{1}$ is the covariance matrix of the data inferred from the masked sky maps. We have subtracted the global mean of unmasked pixels from each map before performing ILC i.e $\langle S\rangle=0$.

\section{Results}

Applying the above mentioned component separation method to half ring ${ }^{2}$ maps, we obtain the half ring ALP distortion maps using 70 - $545 \mathrm{GHz}$ sky maps of Planck, all smoothed to a common angular resolutions (15 or 20 arcmin) and combine them to get the half-ring-half-sum (HRHS) and half-ring-half-difference (HRHD) maps. The HRHS map includes both signal and noise, whereas the HRHD gives the noise estimate in the HRHS map.

The HRHS map for sky fraction $45 \%$ is shown in Fig. 1. We plot the 1-D Probability Distribution Function (PDF) in Fig. 3. There are a significant number of pixels above the Gaussian HRHD noise PDF making the HRHS PDF broader with a significant positive tail. All (or most) of the signal is contamination from other components such as CMB, SZ, dust as well as unresolved point sources. As a result this map is only an upper bound on $\alpha$-distortion. For $f_{s k y}=27 \%$ and $f_{s k y}=45 \%$ with 15 arcmin smoothing scale, the $95^{\text {th }}$ percentile upper limits from HRHS maps are $17.3 \times 10^{-6}$ and $18.5 \times 10^{-6}$ respectively. These bounds are conservative upper limits on the ASD signal which include contaminations from instrument noise as well as astrophysical and cosmological signals.

Assuming that the signal is dominated by contamination from other components, we can put an upper limit to the RMS ALPs distortions, $\sigma_{R M S}^{\alpha}$, after removing the noise contribution, $\sigma_{R M S}^{\alpha}=$ $\left(\sigma_{H R H S}^{2}-\sigma_{H R H D}^{2}\right)^{1 / 2}$. The upper limits on the ALPs distortion for different resolutions is shown in Table 1 .

Table 1: $\sigma_{R M S}^{\alpha}$ in units of $10^{-6}$

\begin{tabular}{|c|c|c|c|}
\hline$f_{\text {sky }}$ & $\begin{array}{l}\text { smoothing } \\
\text { scale (in } \\
\text { arcmin) }\end{array}$ & $\begin{array}{l}70-545 \quad(\mathrm{GHz}) \\
\sigma_{R M S}^{\alpha}\end{array}$ & $\begin{array}{l}100-545 \\
(\mathrm{GHz}) \sigma_{R M S}^{\alpha}\end{array}$ \\
\hline \multirow[t]{2}{*}{0.27} & 15 & 10.3 & 10.7 \\
\hline & 20 & 7.9 & 8.1 \\
\hline \multirow[t]{2}{*}{0.45} & 15 & 10.6 & 11.1 \\
\hline & 20 & 8.1 & 8.3 \\
\hline
\end{tabular}

These constraints are obtained by only using the frequency spectrum of the ASD signal and without assuming any model of electron density and magnetic field. However, in order to convert these constraints into constraints on photon-axion coupling strength $g_{\gamma a}$, we need a model of the turbulent electron density and magnetic field of our Galaxy. A further complication is that these constraints are for the fluctuations of the ASD (RMS) i.e. fluctuation of probability of conversion defined in Eq. (2.4) and not the average ASD. With the assumption that the fluctuations in the signal are of the same order as its average value [36], we can translate the $1-\sigma_{R M S}^{\alpha}$ bound on ASD into a bound on $g_{\gamma \mathrm{a}}$. For a typical $\sigma_{R M S}^{\alpha} \lesssim 10^{-5}$ translates into a bound on photon axion coupling of $g_{\gamma \mathrm{a}} \lesssim 10^{-9} \mathrm{GeV}^{-1}$ for Milky Way using Eq. (2.4). The $1-\sigma_{R M S}^{\alpha}$ bound obtained from the CMB maps on $P(\gamma \rightarrow a)$ does not alter with the change in the magnetic field model and depends only on the

\footnotetext{
1 The angular bracket denotes average over pixels.

2 Half ring maps are the Planck maps produced from the first (or second) half of the pointing period.
} 


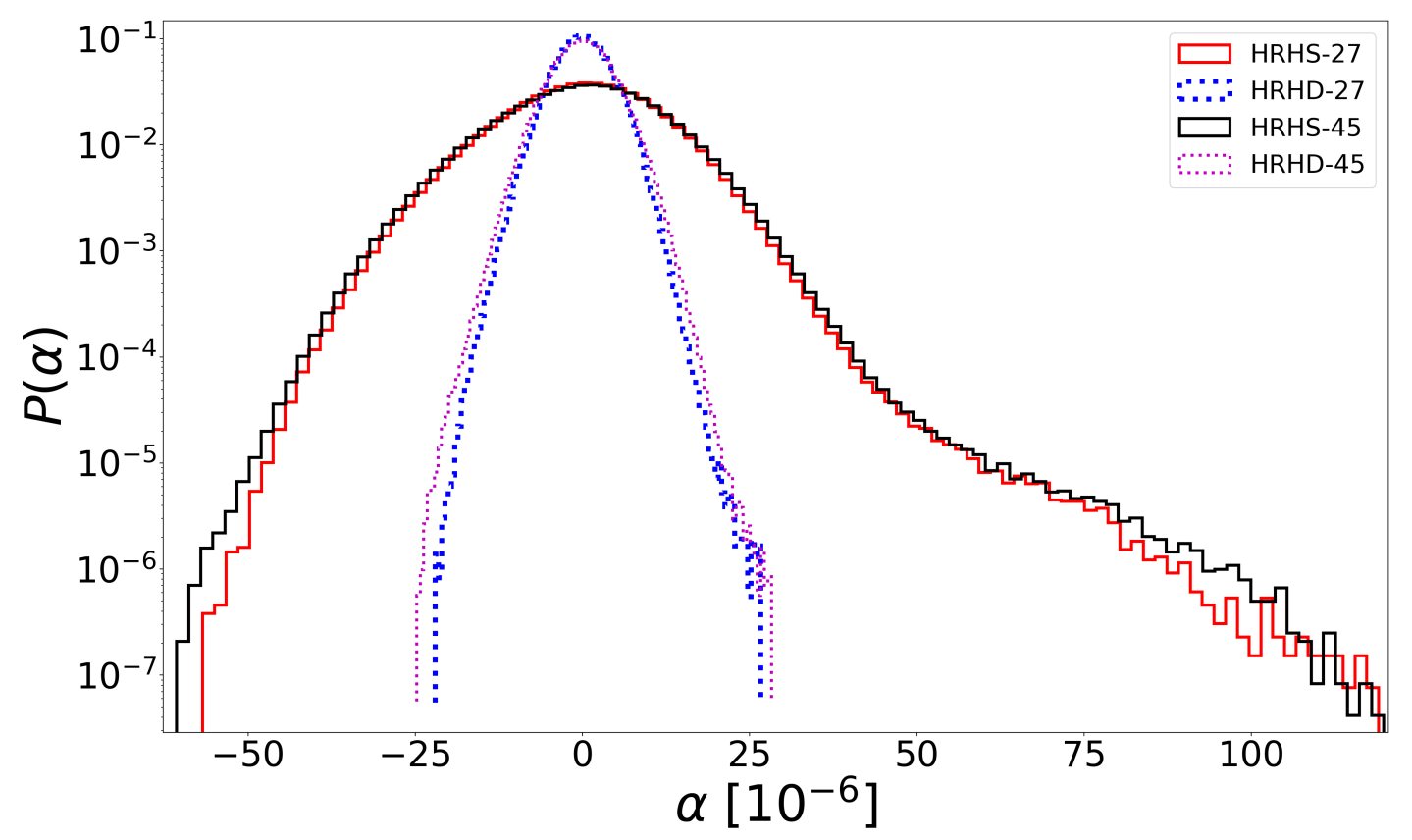

Figure 3: The 1-D probability distribution function of the masked HRHS and HRHD maps are depicted for two $f_{s k y}$ values. The HRHS maps contain contamination from other components. The HRHD maps contain only noise and are close to the expected Gaussian distribution.

spectral shape of the ASD signal. However, in order to relate the constraints on $P(\gamma \rightarrow a)$ with $g_{\gamma \mathrm{a}}$, we require a model of the magnetic field. The measurements of the galactic magnetic field are made with about $25 \%-30 \%$ error-bars $[36,37]$. So, our bound of $g_{\gamma \text { a }}$ can vary within the uncertainty of the magnetic field. The current particle physics bounds from the CERN ALP Solar Telescope (CAST) is $g_{\gamma \mathrm{a}}<6.6 \times 10^{-11} \mathrm{GeV}^{-1}$ at $95 \%$ C.L. [27]. The bound obtained from the Planck data provides an independent but a weaker bound than the current bound from CAST.

We can also calculate the angular power spectrum of the ASD map providing upper bounds on ALPs distortion fluctuations on different angular scales. We calculate the cross-power spectrum of the half-ring maps using PolSpice [56, 57] with the mask apodised by a 30 arcmin Gaussian [25]. The power spectrum $D_{l}=l(l+1) \hat{C}_{l} / 2 \pi$, where $\hat{C}_{l}=\sum_{m} \alpha_{l m}^{H R 1} \alpha_{l m}^{* H R 2} /(2 l+1)$ and, $\alpha_{l m}$ is the spherical harmonic transform of the ALP distortion map, is shown (after correction for the effects of mask and beam[57-60]) in Fig. 4. The Gaussian error-bars on $D_{l}$ are the analytical estimates obtained using PolSpice [56, 57].

\section{Conclusion}

In this paper, we provide the first observational constraints on the non-resonant photon-ALP conversion (or Axion Spectral Distortion ( ASD)) using Planck data. The ASD can be created in the Milky Way when the CMB photons travel through the turbulent magnetic field in the galactic halo and get converted to light spin-0 particles such as light axion particles $\left(m_{a}<<E_{C M B}\right)$ or light scalars. Since both the stochastic magnetic field and the electron density have large fluctuations, the induced spectral distortions will vary on the sky creating a spatially fluctuating unpolarized spectral distortions. The unpolarized ASD has a unique spectral shape different from CMB and other known spectral distortions signal such as $y$-type distortions.

Using six frequency channels $(70,100,143,217,353,545) \mathrm{GHz}$ of the Planck satellite, we obtain the sky-map for the ASD using the ILC algorithm. In order to minimize the contaminations, we mask the most contaminated sky with two different masks having the unmasked sky fractions $27 \%$ 


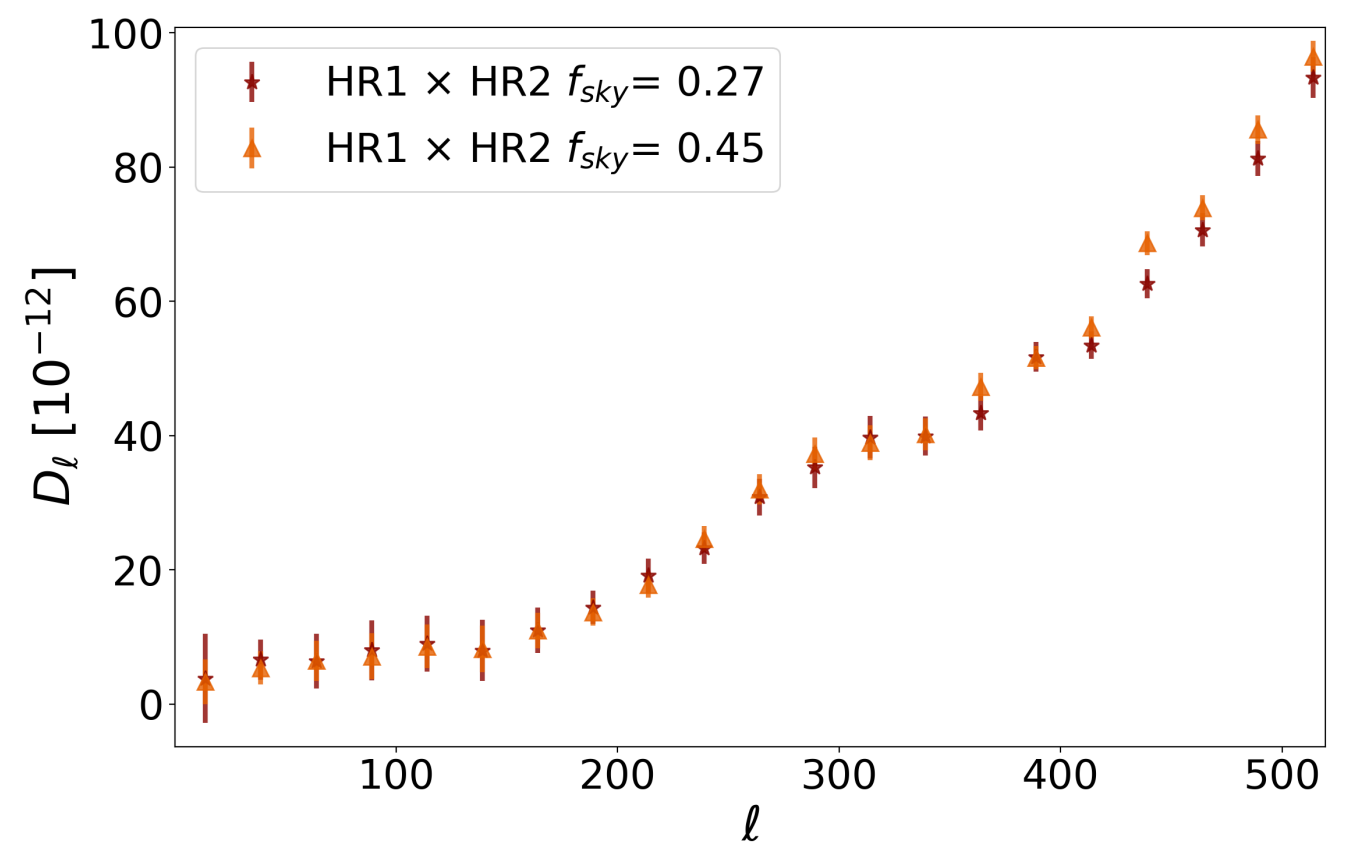

(a)

Figure 4: The angular power spectrum $D_{l} \equiv l(l+1) \hat{C}_{l} / 2 \pi$ for $H R 1 \times H R 2$ for two different masked sky mask $\left(f_{s k y}=0.27\right.$ and 0.45$)$.

and $45 \%$. The sky map of the ASD is shown in Fig. 1. These maps are dominated by the residual contaminations from other components. Hence, we can only provide upper limits on the ASD shown in Table 1. These are robust constraints on the fluctuation of probability of conversion from photon to axion. We expect the fluctuations of ASD on the sky to be of the order unity since the stochastic magnetic fields as well as the electron density have large fluctuations in our own Galaxy as well as outside it where we expect larger contributions from the directions of the nearby voids and smaller distortions from other directions. We can therefore assume that average distortions from our own Galaxy and nearby voids is of the same order of magnitude as the RMS fluctuations. Under this assumption the upper limit of $10.6 \times 10^{-6}$ can be translated into combined limits on $g_{\gamma a} B_{T}$ using Eq. (2.4). A future data-driven model of the galactic magnetic field in future will allow making more precise statements. Future experiments such as Simons Observatory [61], Simons Array [62], Adv-ACT [63], SPT-3G [62] and proposed missions like CMB-S4 [64], LiteBIRD [65], CMB-Bharat, PIXIE [66] and PICO [67] will improve these constraints significantly. Future CMB experiments having more frequency channels will play a crutial role in removing the contamination from CMB fluctuations and the galactic foregrounds. For Planck number of frequency channels is the main limitation. In particular we are limited by foreground contamination and not by the detector noise. Lower detector noise and higher angular resolution than Planck will also play an important role to reduce the noise in the recovered ASD maps and improving the constraints on $g_{\gamma a}$. The polarization data of Planck (and also of ground based experiments), is capable of imposing constraints on the resonant photon-axion conversion [18], which can directly constrain the photon-axion coupling $g_{\gamma a}$ given a model of the Galactic magnetic field. We will address the polarized ASD in a future analysis by using the Planck-2018 polarization data.

Acknowledgement SM would like to thank Nick Battaglia, Siguard Naess, Joseph Silk and David Spergel for useful discussions. The Flatiron Institute is supported by the Simons Foundation. The work of SM and BDW is also supported by the Labex ILP (reference ANR-10-LABX-63) part of the Idex SUPER, and received financial state aid managed by the Agence Nationale de la Recherche, as part of the programme Investissements d'avenir under the reference ANR-11-IDEX-0004-02. The 
work of BDW is also supported by the grant ANR-16-CE23-0002. RK was supported by SERB grant no. ECR/2015/000078 from Science and Engineering Research Board, Department of Science and Technology, Govt. of India and MPG-DST partner group between Max Planck Institute for Astrophysics, Garching and Tata Institute for Fundamental Research, Mumbai funded by Max Planck Gesellschaft.

\section{References}

[1] Y. B. Zeldovich and R. A. Sunyaev, The Interaction of Matter and Radiation in a Hot-Model Universe, ApSS 4 (1969) 301.

[2] R. A. Sunyaev and Y. B. Zeldovich, The interaction of matter and radiation in the hot model of the Universe, II, ApSS 7 (1970) 20.

[3] J. Chluba and R. A. Sunyaev, The evolution of CMB spectral distortions in the early Universe, MNRAS 419 (2012) 1294.

[4] J. Chluba, R. Khatri and R. A. Sunyaev, $C M B$ at $2 \times 2$ order: the dissipation of primordial acoustic waves and the observable part of the associated energy release, MNRAS 425 (2012) 1129 [1202.0057].

[5] R. Khatri and R. A. Sunyaev, Creation of the CMB spectrum: precise analytic solutions for the blackbody photosphere, JCAP 6 (2012) 038 [1203.2601].

[6] R. Khatri, R. A. Sunyaev and J. Chluba, Mixing of blackbodies: entropy production and dissipation of sound waves in the early Universe, A\&A 543 (2012) A136 [1205.2871].

[7] R. Khatri and R. A. Sunyaev, Beyond $y$ and $\mu$ : the shape of the CMB spectral distortions in the intermediate epoch, $1.5 \times 10^{4} \lesssim z \lesssim 2 \times 10^{5}$, JCAP 9 (2012) 016 [1207.6654].

[8] R. A. Sunyaev and R. Khatri, Unavoidable CMB Spectral Features and Blackbody Photosphere of Our Universe, International Journal of Modern Physics D 22 (2013) 1330014 [1302.6553].

[9] J. Chluba, Which spectral distortions does $\Lambda$ CDM actually predict?, MNRAS 460 (2016) 227 [1603.02496].

[10] J. C. Hill, N. Battaglia, J. Chluba, S. Ferraro, E. Schaan and D. N. Spergel, Taking the Universe's Temperature with Spectral Distortions of the Cosmic Microwave Background, Physical Review Letters 115 (2015) 261301 [1507.01583].

[11] R. Emami, E. Dimastrogiovanni, J. Chluba and M. Kamionkowski, Probing the scale dependence of non-Gaussianity with spectral distortions of the cosmic microwave background, Phys.Rev.D 91 (2015) 123531 [1504.00675].

[12] M. Hasselfield et al., The Atacama Cosmology Telescope: Sunyaev-Zel'dovich selected galaxyclusters at 148 GHz from three seasons of data, JCAP 1307 (2013) 008 [1301.0816].

[13] SPT collaboration, Galaxy Clusters Discovered via the Sunyaev-Zel'dovich Effect in the 2500-square-degree SPT-SZ survey, Astrophys. J. Suppl. 216 (2015) 27 [1409.0850].

[14] Planck collaboration, Planck 2015 results. XXVII. The Second Planck Catalogue of Sunyaev-Zeldovich Sources, Astron. Astrophys. 594 (2016) A27 [1502.01598].

[15] Z. Staniszewski et al., Galaxy clusters discovered with a Sunyaev-Zel'dovich effect survey, Astrophys. J. 701 (2009) 32 [0810.1578].

[16] M. Zemcov et al., First detection of the Sunyaev Zel'dovich effect increment at $\lambda<650 \mu m$, A\&A 518 (2010) L16 [1005.3824].

[17] Planck Collaboration, P. A. R. Ade, N. Aghanim, M. Arnaud, M. Ashdown, F. Atrio-Barandela et al., Planck intermediate results. VIII. Filaments between interacting clusters, A\&A 550 (2013) A134 [1208.5911].

[18] S. Mukherjee, R. Khatri and B. D. Wandelt, Polarized anisotropic spectral distortions of the CMB: Galactic and extragalactic constraints on photon-axion conversion, JCAP 1804 (2018) 045 [1801.09701].

[19] D. J. Fixsen, E. S. Cheng, J. M. Gales, J. C. Mather, R. A. Shafer and E. L. Wright, The Cosmic Microwave Background Spectrum from the Full COBE FIRAS Data Set, ApJ 473 (1996) 576. 
[20] D. J. Fixsen, G. Hinshaw, C. L. Bennett and J. C. Mather, The Spectrum of the Cosmic Microwave Background Anisotropy from the Combined COBE FIRAS and DMR Observations, ApJ 486 (1997) 623 [astro-ph/9704176].

[21] D. J. Fixsen and J. C. Mather, The Spectral Results of the Far-Infrared Absolute Spectrophotometer Instrument on COBE, ApJ 581 (2002) 817.

[22] D. J. Fixsen, The Temperature of the Cosmic Microwave Background, ApJ 707 (2009) 916 [0911.1955].

[23] R. Khatri and R. Sunyaev, Limits on the fluctuating part of y-type distortion monopole from Planck and SPT results, JCAP 8 (2015) 013 [1505.00781].

[24] E. Pajer and M. Zaldarriaga, A hydrodynamical approach to CMB $\mu$-distortion from primordial perturbations, JCAP 1302 (2013) 036 [1206.4479].

[25] R. Khatri and R. Sunyaev, Constraints on $\mu$-distortion fluctuations and primordial non-Gaussianity from Planck data, JCAP 9 (2015) 026 [1507.05615].

[26] A. Arvanitaki, S. Dimopoulos, S. Dubovsky, N. Kaloper and J. March-Russell, String Axiverse, Phys. Rev. D81 (2010) 123530 [0905.4720].

[27] CAST collaboration, New CAST Limit on the Axion-Photon Interaction, Nature Phys. 13 (2017) 584 [1705.02290].

[28] ALPS II collaboration, Any Light Particle Search II - Status Overview, in Proceedings, 11th Patras Workshop on Axions, WIMPs and WISPs (Axion-WIMP 2015): Zaragoza, Spain, June 22-26, 2015, pp. 31-34, 2015, 1509.02070, DOI.

[29] MADMAX Working Group collaboration, MADMAX: A new Dark Matter Axion Search using a Dielectric Haloscope, in Proceedings, 12th Patras Workshop on Axions, WIMPs and WISPs (PATRAS 2016): Jeju Island, South Korea, June 20-24, 2016, pp. 94-97, 2017, 1611.04549, DOI.

[30] S. J. Asztalos, G. Carosi, C. Hagmann, D. Kinion, K. van Bibber, M. Hotz et al., SQUID-Based Microwave Cavity Search for Dark-Matter Axions, Phys.Rev.Lett 104 (2010) 041301 [0910.5914].

[31] D. Budker, P. W. Graham, M. Ledbetter, S. Rajendran and A. O. Sushkov, Proposal for a Cosmic Axion Spin Precession Experiment (CASPEr), Physical Review X 4 (2014) 021030 [1306.6089].

[32] M. Tegmark and G. Efstathiou, A method for subtracting foregrounds from multi-frequency cmb sky maps, Mon. Not. Roy. Astron. Soc. 281 (1996) 1297 [astro-ph/9507009].

[33] G. Raffelt and L. Stodolsky, Mixing of the Photon with Low Mass Particles, Phys. Rev. D37 (1988) 1237.

[34] G. G. Raffelt, Particle Physics From Stars, Annual Review of Nuclear and Particle Science 49 (1999) 163 [hep-ph/9903472].

[35] G. G. Raffelt, Stars as laboratories for fundamental physics : the astrophysics of neutrinos, axions, and other weakly interacting particles. University of Chicago Press, 1996.

[36] R. Jansson and G. R. Farrar, A New Model of the Galactic Magnetic Field, ApJ 757 (2012) 14 [1204.3662].

[37] R. Jansson and G. R. Farrar, The Galactic Magnetic Field, ApJ 761 (2012) L11 [1210.7820].

[38] H. Böhringer, G. Chon and P. P. Kronberg, The Cosmic Large-Scale Structure in X-rays (CLASSIX) Cluster Survey I: Probing galaxy cluster magnetic fields with line of sight rotation measures, Astron. Astrophys. 596 (2016) A22 [1610.02887].

[39] C. L. Bennett, D. Larson, J. L. Weiland, N. Jarosik, G. Hinshaw, N. Odegard et al., Nine-year Wilkinson Microwave Anisotropy Probe (WMAP) Observations: Final Maps and Results, ApJS 208 (2013) 20 [1212.5225].

[40] Planck Collaboration, R. Adam, P. A. R. Ade, N. Aghanim, Y. Akrami, M. I. R. Alves et al., Planck 2015 results. I. Overview of products and scientific results, A\&SA 594 (2016) A1 [1502.01582].

[41] H. K. Eriksen et al., CMB component separation by parameter estimation, Astrophys. J. 641 (2006) 665 [astro-ph/0508268]. 
[42] J. Delabrouille, J. F. Cardoso, M. L. Jeune, M. Betoule, G. Fay and F. Guilloux, A full sky, low foreground, high resolution CMB map from WMAP, Astron. Astrophys. 493 (2009) 835 [0807.0773].

[43] K. K. Rogers, H. V. Peiris, B. Leistedt, J. D. McEwen and A. Pontzen, Spin-SILC: CMB polarization component separation with spin wavelets, Mon. Not. Roy. Astron. Soc. 463 (2016) 2310 [1605.01417].

[44] J. Delabrouille, J. F. Cardoso and G. Patanchon, Multi-detector multi-component spectral matching and applications for CMB data analysis, Mon. Not. Roy. Astron. Soc. 346 (2003) 1089 [astro-ph/0211504].

[45] F. Vansyngel, B. D. Wandelt, J.-F. Cardoso and K. Benabed, Semi-blind Bayesian inference of CMB map and power spectrum, Astron. Astrophys. 588 (2016) A113 [1409.0858].

[46] H. K. Eriksen, A. J. Banday, K. M. Gorski and P. B. Lilje, Foreground removal by an internal linear combination method: Limitations and implications, Astrophys. J. 612 (2004) 633 [astro-ph/0403098].

[47] G. Hurier, S. R. Hildebrandt and J. F. Macias-Perez, MILCA: A Maximum Internal Linear Component Analysis for the extraction of spectral emissions, Astron. Astrophys. 558 (2013) A118 [1007.1149].

[48] M. Remazeilles, J. Delabrouille and J.-F. Cardoso, CMB and SZ effect separation with constrained Internal Linear Combinations, MNRAS 410 (2011) 2481 [1006.5599].

[49] R. Khatri, Linearized iterative least-squares (LIL): a parameter-fitting algorithm for component separation in multifrequency cosmic microwave background experiments such as Planck, Mon. Not. Roy. Astron. Soc. 451 (2015) 3321 [1410.7396].

[50] R. Khatri, Data driven foreground clustering approach to component separation in multifrequency CMB experiments: A new Planck CMB map, 1808.05224.

[51] C. L. Bennett, M. Halpern, G. Hinshaw, N. Jarosik, A. Kogut, M. Limon et al., First-Year Wilkinson Microwave Anisotropy Probe (WMAP) Observations: Preliminary Maps and Basic Results, ApJS 148 (2003) 1.

[52] R. Khatri. http://theory.tifr.res.in/ khatri/muresults/, 2015.

[53] PlancK collaboration, Planck 2015 results. VIII. High Frequency Instrument data processing: Calibration and maps, Astron. Astrophys. 594 (2016) A8 [1502.01587].

[54] Planck Collaboration, "Planck Legacy Archive." http://pla.esac.esa.int/pla, 2015.

[55] Planck Collaboration, P. A. R. Ade, N. Aghanim, C. Armitage-Caplan, M. Arnaud, M. Ashdown et al., Planck 2013 results. IX. HFI spectral response, A\&A 571 (2014) A9 [1303.5070].

[56] G. Efstathiou, Myths and truths concerning estimation of power spectra: the case for a hybrid estimator, MNRAS 349 (2004) 603 [astro-ph/0307515].

[57] A. Challinor, G. Chon, S. Colombi, E. Hivon, S. Prunet and I. Szapudi, "PolSpice." http://www2.iap.fr/users/hivon/software/PolSpice/.

[58] E. Hivon, K. M. Gorski, C. B. Netterfield, B. P. Crill, S. Prunet and F. Hansen, Master of the cosmic microwave background anisotropy power spectrum: a fast method for statistical analysis of large and complex cosmic microwave background data sets, Astrophys. J. 567 (2002) 2 [astro-ph/0105302].

[59] I. Szapudi, S. Prunet, D. Pogosyan, A. S. Szalay and J. R. Bond, Fast Cosmic Microwave Background Analyses via Correlation Functions, ApJL 548 (2001) L115.

[60] G. Chon, A. Challinor, S. Prunet, E. Hivon and I. Szapudi, Fast estimation of polarization power spectra using correlation functions, MNRAS 350 (2004) 914 [astro-ph/0303414].

[61] N. Galitzki, A. Ali, K. S. Arnold, P. C. Ashton, J. E. Austermann, C. Baccigalupi et al., The Simons Observatory: Instrument Overview, ArXiv e-prints (2018) [1808.04493].

[62] A. Suzuki, P. Ade, Y. Akiba, C. Aleman, K. Arnold, C. Baccigalupi et al., The Polarbear-2 and the Simons Array Experiments, Journal of Low Temperature Physics 184 (2016) 805 [1512.07299].

[63] R. J. Thornton, P. A. R. Ade, S. Aiola, F. E. Angilè, M. Amiri, J. A. Beall et al., The Atacama Cosmology Telescope: The Polarization-sensitive ACTPol Instrument, ApJS 227 (2016) 21 [1605.06569].

[64] CMB-S4 collaboration, CMB-S4 Science Book, First Edition, 1610.02743. 
[65] T. Matsumura et al., LiteBIRD: Mission Overview and Focal Plane Layout, J. Low. Temp. Phys. 184 (2016) 824.

[66] A. J. Kogut, D. T. Chuss, J. L. Dotson, E. Dwek, D. J. Fixsen, M. Halpern et al., The Primordial Inflation Explorer (PIXIE), in American Astronomical Society Meeting Abstracts \#223, vol. 223 of American Astronomical Society Meeting Abstracts, p. 439.01, Jan., 2014.

[67] K. Young, M. Alvarez, N. Battaglia, J. Bock, J. Borrill, D. Chuss et al., Optical Design of PICO, a Concept for a Space Mission to Probe Inflation and Cosmic Origins, ArXiv e-prints (2018) [1808.01369]. 